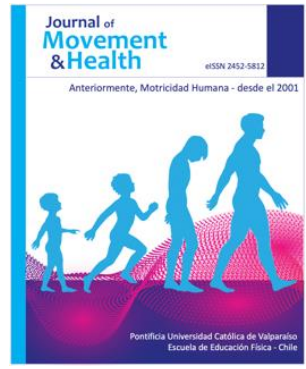

eISSN: $2452-5812$

http://jmh.pucv.cl/

Recibido: $19 / 08 / 2020$

Aceptado: 26/10/2020

Disponible: $11 / 11 / 2020$

Publicado: 01/01/2021

\section{Estudio de casos}

\section{Afectividad de un niño con trastorno del espectro autista en el contexto escolar y familiar: estudio de un caso}

The affectivity of a child with autism spectrum disorder in the school and family context: study of a case

\author{
Campos-Campos, $\mathbf{K}^{1,2}$ \\ Correspondencia ${ }^{\varpi}$ \\ Kevin Isaías Campos-Campos \\ Postgrado Universidad San Sebastián, Concepción, Chile. \\ kevincamposcampos@gmail.com
}

\title{
Resumen
}

Objetivo: Conocer las características socioafectivas de un estudiante diagnosticado con trastorno del espectro autista (TEA) en el contexto escolar y familiar. Métodos: Se utilizó un enfoque cualitativo con un diseño fenomenológico. Estudio de caso: participó del estudio un niño de 12 años diagnosticado con TEA perteneciente a un colegio de educación regular. Se aplicó una entrevista semiestructurada de 14 preguntas distribuidas en dos categorías (social y afectiva). El análisis de datos cualitativos se realizó a través del programa Atlas.ti ${ }^{\circledR} 8$ para Windows, mediante codificación de líneas de respuesta. Resultados: El estudiante presenta mayor afectividad en el contexto familiar. Si bien la relación con sus profesores y compañeros en el establecimiento educacional es positiva, manifiesta que el colegio lo estresa y los profesores de más edad generan un disgusto en él. Conclusión: Se concluye que el niño siente mayor comprensión por los profesores jóvenes. La estructura de largas horas que mantiene el colegio genera un estrés y cansancio emocional.

Palabras clave: trastornos del espectro autista; afectividad; familia; escolar.

\begin{abstract}
Objective: To know the socio-affective characteristics of a student diagnosed with autism spectrum disorder (ASD) in the school and family context. Methods: A qualitative approach with a phenomenological design was used. Case study: a 12-year-old boy diagnosed with ASD from a regular education school participated in the study. A semi-structured interview of 14 questions distributed in two categories (social and affective) was applied. Qualitative data analysis was performed using the Atlas.ti ${ }^{\circledR}$ 8 program for Windows, using response line coding. Results: The student shows greater affectivity in the family context. Although the relationship with his teachers and schoolmates in the educational establishment is positive, he says the school stresses him and the older teachers generate displeasure in him. Conclusion: It was concluded that the child feels more understanding by young teachers and that the structure of long hours that school has generated emotional stress and fatigue in him.
\end{abstract}

Keywords: autism spectrum disorders; affectivity; family; school. 


\section{Puntos destacables}

- El estudiante no genera lazos afectivos con sus compañeros y profesores, a pesar de mantener buena relación.

- Los profesores de más edad generan estrés al estudiante debido a la poca comprensión de su condición.

- La estructura de largas horas que mantiene el colegio genera tensión emocional provocando frustración e irritabilidad.

- El estudiante manifiesta acciones de afecto hacia su entorno familiar.

\section{Introducción}

El Ministerio de Educación (Chile), en su Decreto $\mathrm{N}^{\circ} 170 / 2009^{1}$, establece que el trastorno del espectro autista (TEA) "consiste en una alteración cualitativa de un conjunto de capacidades referidas a la interacción social, la comunicación y la flexibilidad mental, que pueden variar en función de la etapa del desarrollo, la edad y el nivel intelectual de la persona que lo presenta." (p.29). Sin embargo, la definición más actualizada, utilizada universalmente para diagnosticar a un estudiante con TEA, se basa en el Manual Diagnóstico y Estadístico de los Trastornos Mentales (DSM-V), el cual señala que el TEA se caracteriza por las deficiencias persistentes en la comunicación e interacción social en diversos contextos y por la presencia de patrones restrictivos y repetitivos de comportamiento, intereses o actividades ${ }^{2}$.

El estudio de los trastornos del espectro autista dentro del ámbito educacional cumple un rol fundamental para el conocimiento y comprensión de esta condición, pues en nuestro país, se ha evidenciado un alto aumento en la prevalencia de TEA en niños chilenos. Aunque no existe un registro exacto del diagnóstico, en el año 2011 el Ministerio de Salud (MINSAL) establece que la cantidad aproximada de personas con TEA en Chile sería de 2.156 niños $^{3}$. Actualmente, según las predicciones mundiales llevadas al contexto nacional, se estima que un $1 \%$ de la población presenta TEA ${ }^{4}$. Esto ha generado un gran desafío para profesionales de la educación en el marco de la inclusión escolar, con el fin de lograr una intervención temprana y especializada en los aspectos socioafectivos de los estudiantes.

El vínculo afectivo se define como la atracción que siente un sujeto por otro, determinando el comportamiento social de cada individuo 5 . En ese sentido, es primordial comprender desde el ámbito educativo los sentimientos del estudiante, debido a la alteración social presente en niños con TEA, dificultando la construcción de lazos afectivos con sus familiares y/o profesores, provocando conductas solitarias incluso en sus tiempos libres (recreo). Sin embargo, una intervención temprana y especializada, puede favorecer al compromiso afectivo y, de esta forma, al desarrollo y funcionamiento cerebral lo que conlleva, además, a buenos resultados en el proceso de enseñanza-aprendizaje ${ }^{5-10}$.

A ello se suma la importancia de considerar, cuidadosamente, las emociones en el desarrollo infantil, ya que son respuestas psicofisiológicas a través de las cuales nos adaptamos y respondemos a diversas situaciones que podemos percibir de distinta manera ${ }^{11}$. Son tan complejas y están presentes en todas las personas, que no podemos excluir a nadie del mundo emocional en el que estamos inmersos ${ }^{12}$.

A raíz de lo anterior radica la importancia del profesor, ya que es él quien puede abrir la puerta al cerrado mundo del niño ${ }^{13,14}$. Además, es el encargado, en su papel formador, de entregar habilidades para la vida, lo que es de gran ayuda en estudiantes con dicho diagnóstico ${ }^{15}$. Es por eso que el rol del profesor/a es trascendental, ya que va a desempeñar uno de los papeles más exigentes de todos los que tratan de alguna forma a las personas con TEA.

En ese sentido, el siguiente estudio brinda información relevante para comprender las emociones que pueden presentar niños/as con TEA, generando mayor comprensión tanto para los profesionales de educación como para familiares que recién se están incluyendo al mundo del TEA. Por lo tanto, el objetivo de la investigación fue conocer las características socioafectivas de un estudiante diagnosticado con TEA en el contexto escolar y familiar. 


\section{Métodos}

Se llevó a cabo un estudio de caso de enfoque cualitativo, de diseño fenomenológico. Forma parte del estudio, un niño de 12 años diagnosticado con TEA, según diagnóstico DSM-V ${ }^{2}$, perteneciente a un curso de $6^{\circ}$ básico de un Colegio de la comuna de Concepción, Chile. Es importante destacar que el estudiante ha estado en aula regular durante toda su trayectoria estudiantil.

\section{Procedimientos}

Para conseguir la entrevista con el evaluado, se planteó la iniciativa a la madre del estudiante explicando los objetivos del proyecto de investigación, su relevancia social, entendiendo el desconocimiento del TEA en el contexto educativo, y su importancia para los profesionales de la educación. Para ello, se hizo firmar un consentimiento informado como representante del menor de edad. Luego de conseguir la autorización de la madre, se aplicó una entrevista semiestructurada, la cual consiste en introducir preguntas adicionales para precisar conceptos u obtener mayor información sobre los temas deseados, teniendo como ventaja la posibilidad de adaptarse a los sujetos ${ }^{16,17}$. Esta se llevó a cabo en el hogar del estudiante para asegurar la comodidad del evaluado junto con su familia.

\section{Entrevista}

La entrevista (tabla 1) fue confeccionada de forma independiente por el evaluador, y validada por tres especialistas con postgrado $(n=1$ postdoctorado, $n=1$ doctorado $y=1$ magíster) en el área de Actividad Física Adaptada e Inclusión Escolar. La cual contiene 14 preguntas distribuidas en dos categorías; (1) social y (2) afectiva.

Tabla 1. Entrevista

\begin{tabular}{cl}
\hline $\mathbf{N}^{\circ}$ & \multicolumn{1}{c}{ Preguntas } \\
\hline 1. & ¿Cómo te sientes con el trato que recibes de las personas en tu colegio? \\
2. ¿Qué reacción tienen los profesores de asignatura cuando quieres expresar alguna opinión o idea? \\
3. ¿Te acercas a algún profesor para conversar sobre alguna inquietud o un tema en particular? \\
4. ¿Sientes el apoyo por parte de los docentes de tu colegio? \\
5. ¿Hay algún profesor o profesora con que sientas mayor afinidad? \\
6. ¿Qué haces en los momentos de descanso o entretenimiento (recreos)? \\
7. ¿Tus compañeros te incluyen en las conversaciones y/o juegos que realizan de forma grupal? \\
8. ¿Te gusta la metodología que utiliza el profesor de Educación Física para realizar sus clases? \\
9. ¿Cómo te sientes cuando vas al colegio? ¿Te gusta? \\
10. ¿Qué prefieres, asistir al colegio o quedarte en casa? ¿Por qué? \\
11. ¿Sientes tranquilidad al momento de estar en casa junto con tu familia? \\
12. ¿Cuándo te sientes triste, conversas con algún integrante de tu familia? ¿Y en otros momentos, te \\
gusta conversar con algún familiar? \\
13. ¿Sientes que tu familia te entrega cariño? ¿Y tú, les demuestras tu afecto? \\
14. ¿Cómo crees que tu familia te demuestra el cariño?
\end{tabular}

La recolección de las informaciones se llevó a cabo por el evaluador (investigador) en un ambiente restricto donde se resguardó el cuidado de retirar todo objeto presente en el espacio con la finalidad de reducir un desvío de la atención por el evaluado (estudiante). La entrevista fue aplicada de forma verbal y respondida de la misma forma por el evaluado (estudiante). Las respuestas del estudiante fueron grabadas en audio para posteriormente transcribirlas y analizar las transcripciones detallada y literalmente. 


\section{Análisis de la información}

Posterior a la transcripción se utilizó el programa Atlas.ti® 8 para Windows, con la finalidad de generar una bitácora de análisis, cuya función es documentar paso a paso el proceso analítico, donde se realizó una codificación de los resultados cualitativos a través de unidades constantes mediante líneas de cada respuesta, es decir, se identifican propiedades de los datos en cada línea de respuesta y se construyen categorías. Finalmente, los resultados son descritos y analizados en función de cuatro categorías: socialización escolar, afectividad escolar, socialización familiar y afectividad familiar.

\section{Resultados}

\section{Socialización escolar}

Cuatro preguntas de la entrevista fueron enfocadas al análisis de socialización en el contexto escolar (tabla 2). Los resultados del estudiante evaluado demuestran que, es de su interés el expresar sus ideas u opiniones en la sala de clases, sin embargo, dentro de su ímpetu por opinar, no respeta turnos (pedir la palabra), provocando molestias en sus compañeros y profesores. Por otro lado, el estudiante manifiesta que en sus recreos prefiere jugar con aparatos tecnológicos (celular), no suele jugar con sus compañeros porque no lo incluyen en sus juegos y tampoco son de su interés, aunque hay momentos en que comparten gustos (ej.: jugar fútbol) pero prefiere no jugar con sus compañeros ya que los estudiantes mayores no le dan el espacio en el gimnasio del colegio para poder realizar sus juegos con tranquilidad. Además, sus compañeros no lo hacen partícipe de conversaciones, aunque no siente la motivación de participar en ellas.

La jornada extensa de clases genera un estrés en el estudiante, causando en él, un estado de tensión emocional, desencadenando actitudes de frustración e irritabilidad, lo que aumenta el rechazo hacia el establecimiento educacional.

Tabla 2. Preguntas enfocadas al análisis de socialización escolar

\section{Preguntas}

2. ¿Qué reacción tienen los profesores de asignatura cuando quieres expresar alguna opinión o idea?

3. ¿Te acercas a algún profesor para conversar sobre alguna inquietud o un tema en particular?

6. ¿Qué haces en los momentos de descanso o entretenimiento (recreos)?

7. ¿Tus compañeros te incluyen en las conversaciones y/o juegos que realizan de forma grupal?

\section{Afectividad escolar}

Seis preguntas de la entrevista fueron orientadas al análisis de la afectividad escolar (tabla 3). En este aspecto, los resultados manifiestan un disgusto por parte del estudiante hacia los profesores mayores, debido a que se irritan con mayor facilidad, elevando la voz y causando molestias en el estudiante. En consecuencia, destaca el agrado que le genera ser partícipe de clases por parte de profesores jóvenes, entendiendo, además, mejor las instrucciones. Asimismo, hace referencia a un buen trato por parte de los docentes, reconociendo el vínculo que generan con los estudiantes. Sin embargo, no conversa sus problemas con los profesores de asignatura cuando siente tristeza, aunque manifiesta que el apoyo recibido, es el óptimo para él. Igualmente, reconoce el respeto que le tienen sus compañeros, pese a ello, no siente la necesidad de generar lazos afectivos con sus pares. 
Tabla 3. Preguntas enfocadas al análisis de la afectividad escolar en el estudiante

\section{Preguntas}

1. ¿Cómo te sientes con el trato que recibes de las personas en tu colegio?

4. ¿Sientes el apoyo por parte de los docentes de tu colegio?

5. ¿Hay algún profesor o profesora con que sientas mayor afinidad?

8. ¿Te gusta la metodología que utiliza el profesor de Educación Física para realizar sus clases?

9. ¿Cómo te sientes cuando vas al colegio? ¿Te gusta?

10. ¿Qué prefieres, asistir al colegio o quedarte en casa? ¿Por qué?

\section{Socialización familiar}

Una pregunta fue enfocada al análisis de la socialización familiar (tabla 4). El estudiante no siente necesidad de conversar con sus familiares, incluso cuando se siente triste, su comodidad es estar alejado de las personas, preferentemente con aparatos tecnológicos los cuales generan un estado de comodidad y entretención, manteniendo conversaciones con niños en juegos online. Sin embargo, reconoce el gran apoyo recibido por su entorno cercano.

Tabla 4. Pregunta enfocada al análisis de socialización familiar

\section{Preguntas}

12. ¿Cuándo te sientes triste, conversas con algún integrante de tu familia? ¿Y en otros momentos, te gusta conversar con algún familiar?

\section{Afectividad familiar}

Finalmente, tres preguntas fueron orientadas al análisis de la afectividad familiar (tabla 5). A pesar de no sentir la necesidad de conversar con sus familiares, manifiesta un gusto por estar junto a sus seres queridos y sintiendo el afecto positivo que le entregan al comprarle cosas de su interés. En este aspecto el estudiante revela un cariño especial hacia sus familiares, expresando el cariño mediante abrazos y reconociendo el valor de su familia.

Tabla 5. Preguntas enfocadas al análisis de la afectividad familiar en el estudiante

\section{Preguntas}

11. ¿Sientes tranquilidad al momento de estar en casa junto con tu familia?

13. ¿Sientes que tu familia te entrega cariño? ¿Y tú, les demuestras tu afecto?

14. ¿Cómo crees que tu familia te demuestra el cariño?

\section{Discusión}

Los resultados manifiestan un mayor afecto hacia su entorno familiar, expresando sus sentimientos de alegría y sintiendo un ambiente de comodidad, el cual no presenta en el colegio, provocando en él, un disgusto el asistir a un establecimiento educacional donde no siente necesidad de crear lazos con sus compañeros de aula y tampoco generar conversaciones con sus profesores. Viéndose así reflejada una característica propia del TEA en el que se ve afectado el desarrollo y mantenimiento de vínculos sociales y afectivos, incluso el fracaso o la deserción escolar ${ }^{7}$. 
La relación con sus compañeros, si bien no es de carácter negativa, tampoco busca lograr una relación de amistad con sus pares. López y Garcia $^{7}$ establecen que el fracaso en el desarrollo y mantenimiento de vínculos sociales se manifiesta, de forma genérica, a modo de aislamiento social. En ese sentido, Bauminger y $\operatorname{Kasari}^{18}$ realizaron un estudio en donde compararon a 22 niños con autismo y 19 niños con desarrollo típico. Se les pidió que informaran sobre su comprensión y sentimientos de soledad como sobre la calidad de su amistad. Al respecto los resultados muestran que los niños con autismo son más solitarios y tienen una menor comprensión de la relación entre la soledad y la amistad.

Si bien las amistades de niños con TEA y normo típicos favorecen el andamiaje de la interacción social, existen ciertas limitaciones que los docentes deben tener en cuenta, pues los niños con TEA presentan menos oportunidades (muchas veces) para tomar un papel de liderazgo en ciertas actividades, lo que puede provocar un desequilibrio traducido en descompensaciones o bien, aislarse en los recreos ${ }^{7,19,20}$.

En ese sentido, Urrutxi y Velásquez ${ }^{21}$ señalan que los recreos resultan confusos para los niños con TEA, debido a que no poseen una estructura concreta y son espacios con ruido excesivo lo que provocan comportamientos ansiosos. Los establecimientos educacionales deben comprender estas causas y generar ambientes para estimular y desarrollar competencias sociales, ya que los recreos resultan una oportunidad imprescindible para potenciar el área social y motriz, por decir algunas.

Rodríguez, Martín, Carbonero y Ovejero ${ }^{22}$ realizaron una intervención aplicada en el recreo para mejorar las habilidades sociales de un estudiante con TEA de alto funcionamiento mediado por estudiantes sin TEA. La intervención duró 14 sesiones y logró cambios notorios en la disminución de tiempos de soledad e incrementó el tiempo de interacción social, pudiendo ser una estrategia replicable por los profesores para mejorar las habilidades sociales por parte de los estudiantes con TEA de alto funcionamiento, teniendo en consideración las limitaciones existentes ${ }^{19}$.

Los resultados de este estudio demuestran que los profesores de más edad causan desagrado en el estudiante, debido a la poca paciencia y comprensión. Puede ser que los niños con TEA plantean a los profesores considerables desafíos en el manejo de sus necesidades educativas especiales. Diversos autores $^{14,22-24}$ sugieren que esto puede deberse a que los docentes no se encuentran preparados para comprender el TEA.

En el ámbito afectivo, la importancia de este vínculo considera desde la interacción que se establece entre pares hasta la relación vincular entre un docente y un estudiante y, a su vez, en cómo esa relación afectiva facilita el proceso de aprendizaje $\mathrm{e}^{25}$. En este caso, dicha afirmación cobra un protagonismo inconmensurable, dada la importancia de la figura de los docentes, lo que no es diferente en niños que presentan TEA, pues más aún en estos casos y con niños y niñas en esta condición, el docente, en palabras de Hans Asperger "debe mostrarse siempre tranquilo, templado y mantener el control... el maestro tiene que volverse también un poco autista" 26 (p. 137).

Aunque exista la creencia que las personas con TEA no son capaces de mantener ninguna relación afectiva con su entorno ${ }^{27}$, sí pueden mantener relaciones amicales y estrechar lazos de afecto y simpatía ${ }^{28}$, tal como se manifiesta en el presente estudio, donde el sujeto expresa un claro afecto hacia sus familiares, comprendiendo también el cariño recibido por parte de su entorno cercano. Si bien en el contexto escolar el vínculo afectivo se ve disminuido, es una variable que puede ser intervenida y potenciada ${ }^{21,29}$, sin embargo, se necesita el conocimiento por parte de los docentes para entender en mayor profundidad el autismo $^{23}$, entendiendo su complejidad y posibles intervenciones para desarrollar los componentes afectivos que contribuyen a una mejor interacción social.

Dentro de las limitaciones de nuestro estudio se reflejan las restricciones propias de la condición, donde el conocer y comprender las características socioafectivas de un niño con TEA, es complejo. Las respuestas concretas pueden dificultar el análisis de la información, no obstante, los hallazgos de este 
estudio pueden ser un inicio para generar un debate relacionado a este ámbito, especialmente en el ámbito educativo, entendiendo la complejidad y variabilidad de la condición en los estudiantes de distintas edades. Por otro lado, se evidencia una escasa literatura referida a comprender el ámbito socioafectivo de los escolares con TEA, por tanto, es importante generar estudios en el ámbito emocional con la finalidad de generar conocimiento empírico.

\section{Conclusiones}

Esta investigación deja en manifiesto que aún existen establecimientos educacionales que no cuentan con las herramientas necesarias para atender a las necesidades de un niño con TEA. La estructura de largas horas aumenta el estrés, en forma de tensión emocional, provocando frustración e irritabilidad en el estudiante con TEA.

La estimulación del vínculo afectivo a temprana edad podría lograr resultados positivos en niños con TEA. Resulta urgente la preparación docente en la comprensión de esta condición para favorecer a través de los lazos afectivos los resultados de aprendizajes e interacción social, tanto en niños diagnosticados con TEA como para niños sin este diagnóstico.

Esta investigación se suma a la escasa información existente acerca del TEA y sus emociones. Se sugiere indagar sobre la afectividad de los niños con TEA de diferentes edades con el fin de generar orientaciones pedagógicas que permitan orientar a los profesionales de la educación, en el desarrollo y la estimulación de las habilidades sociales a temprana edad.

\section{Referencias}

1. Decreto $\mathrm{N}^{\circ} 170 / 2009$, del 21 de abril, fija normas para determinar los alumnos con Necesidades Educativas Especiales que serán beneficiarios de las subvenciones para Educación Especial. (Biblioteca del Congreso Nacional de Chile, Decreto 170, de 21 de abril del 2010). Accedido: 14 enero, 2020. https://www.leychile.cl/Navegar?idNorma=1012570.

2. American Psychiatric Association. Diagnostic and Statistical Manual of Mental Disorders. Washington, DC: American Psychiatric Association; 2013.

3. MINSAL. Detección y Diagnóstico Oportuno de los Trastornos del Espectro Autista (TEA). Ministerio de Salud. Chile; 2011. Accedido: 08, febrero, 2020. http://www.minsal.cl/portal/url/item/bd81e3a09ab6c3cee040010164012ac2.pdf

4. Lampert MP. Trastorno del Espectro Autista. Epidemiología, aspectos psicosociales, y políticas de apoyo en Chile, España y Reino Unido. Biblioteca del Congreso Nacional de Chile; 2018. Accedido: $\quad 09, \quad$ febrero, 2020. https://obtienearchivo.bcn.cl/obtienearchivo?id=repositorio/10221/25819/1/BCN_Politicas_de apoyo_al_espectro_autista_FINAL.pdf

5. Bustos P. [Síndrome de asperger e inhibición conductual: retraso en la gratificación a través de la estimulación del vínculo afectivo]. Revista de Investigación Educacional Latinoamericana. 2017;54(1), 1-12. DOI: 10.7764/PEL.54.1.2017.6

6. Quijada C. [Espectro autista]. Revista chilena de pediatría. 2008;79(1), 86-91. DOI: 10.4067/S037041062008000700013

7. López S, García C. [La conducta socio-afectiva en el trastorno autista: descripción e intervención $\begin{array}{llr}\text { psicoeducativa]. } & \text { Pensamiento } & \text { Psicológico. }\end{array}$ https://revistas.javerianacali.edu.co/index.php/pensamientopsicologico/article/view/96/286

8. Campos P. Trastornos del espectro autista. México: El Manual Moderno; 2007. 
9. Godoy M, Campoverde B. [Análisis comparativo sobre la afectividad como motivadora del proceso enseñanza-aprendizaje. Casos: Argentina, Colombia y Ecuador]. Sophia. 2016;12(2), 217-231. DOI: 10.18634/sophiaj.12v.2i.227

10. Zalaquett D, Schönstedt M, Angeli M, Herrera C, Moyano A. [Fundamentos de la intervención temprana en niños con trastornos del espectro autista]. Rev Chil Pediatr. 2014;86(2), 126-131. DOI: 10.1016/j.rchipe.2015.04.025

11. Álvarez A, Pérez S. Diseño Experimental de una Plataforma Biométrica Hacia el Análisis de la Afectividad y la Evaluación del Trastorno del Espectro Autista TEA. Argentina; 2018. Accedido: 21, junio, 2020. http://www.cerecon.frm.utn.edu.ar/archives/Plataforma-biometrica-emocionalMendoza-Argentina-020618.pdf

12. Miguel A. [El mundo de las emociones en los autistas. Teoría de la Educación]. Educación y Cultura en la Sociedad de la Información. 2006;7(2), 169-183. https://www.redalyc.org/pdf/2010/201017296011.pdf

13. Wing L. The history of Asperger syndrome. En: Schopler E, Mesibov GB, y Kunce LJ, editors. Asperger Syndrome or High Functioning Autism?. New York: Plenum; 1998. pp. 11-28.

14. Lozano J, Alcaraz S, Bernabéu M. [Competencias emocionales del alumnado con Trastornos del Espectro Autista en un Aula Abierta Específica de Educación Secundaria]. Aula Abierta. 2011;40(1), 15-26. https://dialnet.unirioja.es/servlet/articulo?codigo=3791455

15. Zenteno S, Leal S. [Los afectos desde la experiencia de ser profesor de un estudiante diagnosticado con trastorno espectro autista]. Revista de Investigación Educacional Latinoamericana. 2016;53(1), 1-14. DOI: 10.7764/PEL.53.1.2016.5

16. Hernández R, Fernández C, Baptista P. Metodología de la investigación. 5th. ed. México D.F: McGraw-Hill; 2010.

17. Díaz L, Torruco U, Martínez M, Varela M. [La entrevista, recurso flexible y dinámico]. Investigación en Educación Médica. 2013;2(7), 162-167. DOI: 10.1016/S2007-5057(13)72706-6

18. Bauminger N, Kasari C. [Loneliness and Friendship in High-Functioning Children with Autism]. Child Development. 2000;71(2), 447-456. DOI: 10.1111 / 1467-8624.00156

19. Bauminger N, Solomon M, Aviezer A, Heung K, Brown J, Rogers S. [Friendship in High-functioning Children with Autism Spectrum Disorder: Mixed and Non-mixed Dyads]. Journal of Autism and Developmental Disorders. 2007;38(7), 1211-1229. DOI: 10.1007 / s10803-007-0501-2

20. Grandin T. [Case study: How horses helped a teenager with autism make Friends and learn how towork]. International Journal of Environmental Research and Public Health. 2019;16(13), 1-5. DOI: 10.3390 / ijerph16132325

21. Urrutxi L, Velásquez L. [Estrategias educativas para orientar las necesidades educativas de los estudiantes con syndrome de Asperger en aulas ordinarias]. Revista Electrónica de Investigación en Psicología Educativa. 2011;9(2), 869-892. DOI: 10.25115/ejrep.v9i24.1477

22. Rodríguez J, Martín L, Carbonero MA, Ovejero A. [Peer-mediated intervention for the development of social interaction skills in high-functioning autism spectrum disorder: A pilot study]. Frontiers in Psychology. 2016;7, 1-14. DOI: 10.3389 / fpsyg.2016.01986

23. Schliemann A, Alves M, Duarte E. [Educação física inclusiva e autismo]. Rev Bras Educ Fís Esporte. 2020;34(Esp.);77-6. DOI: 10.11606/issn.1981-4690.v34i0p77-86

24. Sciutto M, Richwine S, Mentrikoski J, Niedzwiecki K. [A qualitative analysis of the school experiences of children with Asperger's Syndrome]. Focus on Autism and Other Developmental Disabilities. 2012;27(3), 177-188. DOI: 10.1177/1088357612450511

25. Bustos P, Díaz A, Castro A, Zapata P, Rodríguez G. [Facilitación del vínculo afectivo en educación básica]. Visiones de la Educación. 2014;12(19):43-51. 
DOI: https://doi.org/10.5027/jmh-Vol18-Issue1(2021)art105

26. Silberman S. NeuroTribes: The Legacy of Autism and The Future of Neurodiversity. España: Plantea S.A; 2015.

27. Hobson R. [The emotional origins of social understanding]. Philosophical Psychology. 1993;6, 227245. DOI: $10.1080 / 09515089308573090$

28. Benites L. [Autismo, familia y calidad de vida]. CULTURA. 2010;24, 1-20. https://dialnet.unirioja.es/servlet/articulo? codigo $=3701024$

29. Daou N, Vener S. Poulson C. [Analysis of three components of affective behavior in children with autism]. Research in Autism Spectrum Disorders. 2014;8(5), 480-501. DOI: 10.1016/j.rasd.2014.01.005

\section{Filiaciones}

${ }^{1}$ Postgrado, Universidad San Sebastián, Concepción, Chile.

${ }^{2}$ Grupo de Investigación en Deporte Adaptado y Paralímpico - GIDEPAUSS, Concepción, Chile.

\section{Declaración de Autoría}

K.C.C elaboró el manuscrito en su totalidad.

\section{Conflicto de interés}

No hay conflicto de interés. 